\title{
Rivaroxaban for patients with AF undergoing cardioversion
}

The oral anticoagulant rivaroxaban is a safe and effective alternative to vitamin $\mathrm{K}$ antagonists for patients with atrial fibrillation (AF) undergoing cardioversion. This finding from the $\mathrm{X}$-VeRT trial was presented at the ESC Congress 2014 and simultaneously published in the European Heart Journal.

Electrical or pharmacological cardioversion can be used to restore sinus rhythm in patients with AF, but a risk of thromboembolic complications exists (stroke rate of 5-7\% in patients not receiving anticoagulation therapy). Oral anticoagulants (apixaban, dabigatran, and rivaroxaban) have been shown to be effective alternatives to vitamin $\mathrm{K}$ antagonists for stroke prevention in patients with AF. X-VeRT is the first prospective trial to be published in which an oral anticoagulant was tested in patients with AF undergoing elective cardioversion.

Investigators enrolled 1,504 patients from 141 centres in 16 countries with

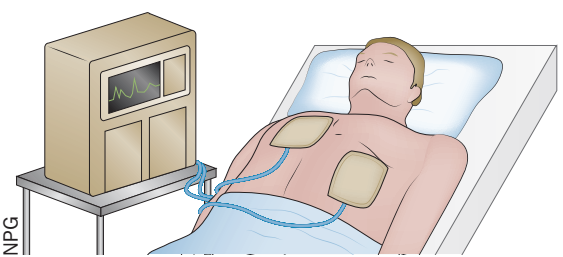

haemodynamically-stable, nonvalvular AF into the open-label study. Patients were randomly allocated to rivaroxaban $(20 \mathrm{mg}$ once daily, or $15 \mathrm{mg}$ if creatinine clearance was $30-49 \mathrm{ml} / \mathrm{min}$ ) or dose-adjusted vitamin $\mathrm{K}$ antagonists. Early or delayed cardioversion (1-5 days or 3-8 weeks after randomization) was performed according to investigator judgement.

The composite primary efficacy end point (stroke, transient ischaemic attack, peripheral embolism, myocardial infarction, and cardiovascular death) occurred in $0.51 \%$ of patients receiving rivaroxaban and $1.02 \%$ of patients receiving a vitamin $\mathrm{K}$ antagonist (risk ratio [RR] 0.50, $95 \%$ CI $0.15-1.73$ ). The primary safety outcome (major bleeding) occurred in $0.6 \%$ and $0.8 \%$ of patients in the rivaroxaban and vitamin $\mathrm{K}$ antagonist groups, respectively (RR 0.76, 95\% CI 0.21-2.67). Time from randomization to cardioversion was significantly shorter in patients receiving rivaroxaban compared with those receiving a vitamin $\mathrm{K}$ antagonist.

The investigators conclude that "rivaroxaban administered de novo, or as ongoing therapy, or as a replacement for vitamin $\mathrm{K}$ antagonists or another anticoagulant agent was associated with thromboembolic and bleeding risks that were low and similar to those observed with vitamin K antagonists". They hope that rivaroxaban "may allow prompter cardioversion" than existing anticoagulation regimens with vitamin $\mathrm{K}$ antagonists.

Gregory B. Lim

Original article Cappato, R. et al. Rivaroxaban vs. vitamin $\mathrm{K}$ antagonists for cardioversion in atrial fibrillation. Eur. Heart J. doi:10.1093/eurheartj/ehu367 